\title{
Comparative Study of Stator Core Composition in Transverse Flux Rotary Machine
}

\author{
Ji-Young Lee*, Seung-Ryul Moon*, Dae-Hyun Koo*, Do-Hyun Kang*, \\ Geun-Ho Lee ${ }^{\dagger}$ and Jung-Pyo Hong**
}

\begin{abstract}
This paper deals with the comparison of magnetic characteristics in transverse flux rotary machine according to different stator core composition with the same rotor. Three different stator designs are considered in the analysis according to the material composition of inner and outer stator cores. Electromotive force (EMF), inductance, torque, and core losses are calculated by threedimensional finite element analysis. Calculated and measured results of back-EMF according to the analysis models in dependency on speed are presented.
\end{abstract}

Keywords: Core losses, Soft magnetic composite, Electromotive force, Transverse flux machine

\section{Introduction}

Recently, soft magnetic composite (SMC) has been developed as an alternative magnetic material to reduce the iron losses in electric machine. Moreover, SMC gives additional degrees of electric machine designing freedom, especially when the magnetic flux has to flow threedimensionally in the magnetic parts. However, SMC exhibits lower saturation flux densities and permeability than silicon steel [1]-[4].

With silicon steel, magnetic circuits are established by stacking magnetic sheets. In these magnetic circuits, the magnetic flux has to flow in parallel to the sheet steel surface. Such configuration with silicon steel exhibits very good magnetic proprieties; however, the geometries of possible magnetic circuits are limited because of the anisotropy of the laminated electrical steel and the manufacturing constraints [1], [3].

This paper attempts to find a good composition with these two representative materials, SMC and silicon steel, to achieve high-power density and to reduce core losses in the transverse flux rotary machine (TFRM) with threedimensional magnetic flux paths. Three analysis models are presented. These three models share an identical rotor; however, three different types of stator are used depending on materials: SMC only, silicon steel only, and a combination of SMC and silicon steel. The conditions of the three analysis models are the same, except for stator core materials. The electromagnetic parameters for each model are computed with three-dimensional finite element analysis

$\dagger \quad$ Corresponding author: Dept. of Automotive Engineering, Kookmin University, Korea. (1gh700@korea.com)

* Dept. of Electric Motor Research Center, Korea Electrotechnology Research Institute, Korea. (jylee@keri.re.kr)

** Dept. of Automotive Engineering, Hanyang University, Korea. (hongjp@hanyang.ac.kr)

Received: April 23, 2010; Accepted: December 15, 2010
(FEA). Electromotive force (EMF), inductance, torque, and core losses are obtained with computation results of the time-domain three-dimensional magnetic fields. The three analysis models are fabricated, and EMFs of each model are measured to confirm the core losses depending on material. The most suitable stator core composition is selected from the analysis and experiment results.

\section{Analysis Models}

\subsection{Permanent Magnet Excited TFRM}

Fig. 1 shows the conceptual configuration of one phase in a permanent magnet (PM)-excited TFRM and the magnetic flux path by current excitation. The PMs on rotor are arranged and magnetized in circumferential direction (Fig. (a)) to concentrate the magnetic flux to the rotor core. SMC is used as rotor core material because it is able to easily form a three-dimensional magnetic flux path; however, the magnetic permeability and saturation flux density of SMC are lower than those of laminated silicon steel.

The one-phase stator consists of a coil and the stator core. The stator core is divided into two parts: inner and outer cores. The inner and outer cores are called yoke and teeth, respectively. The teeth are again divided into upper and lower parts again, and the lower teeth are shifted by a half pole-pitch with respect to upper teeth to create skewing effect. Fig. 1(b) shows flux path on cross-section of "A" in Fig. 1(a) when current flows counterclockwise.

\subsection{Analysis Models}

Three kinds of analysis models are dealt according to the material composition of inner and outer stator cores. Table 
1 shows the three analysis models: SM (SMC core Model), SLM (SMC and Laminated silicon steel core Model), and LM (Laminated silicon steel core Model). The configurations are shown in Fig. 2. The left-side figures in Fig. 2 are the conceptual drawings of three analysis models, and the right-side pictures are their actual objects. For the model SLM, the combination of SMC for inner core and silicon steel for outer core is only considered. The opposite case is omitted because the characteristics can be deduced from the results of the three models. When making teeth of LM model, the original shape is the same as the teeth of SLM model. However, a slit is formed in the teeth of LM model (Fig. 2(c)) to improve the motor characteristics. The effect is explained in experimental results of the next section. The active dimensions of the three models are the same, and the dimensions are shown in Table 2.

Generally, lamination is not modeled to reduce computation time in the FEA process. However, in this paper, the lamination is expressed in the analysis to compare the eddy current loss depending on core state. The stator core is modeled as a solid core when modeling SMC core, whereas it is modeled as layers when modeling laminated silicon steel core. To reduce the number of elements and save computation time, the lamination is expressed with a few layers, not the actual number of laminated sheets, and with insulating boundary condition instead of air gap between layers [5]-[6]. Layers separated by the insulating boundary condition are only electrically isolated, not magnetically or mechanically.

\subsection{Analysis Conditions}

Specifications of the prototype, including magnetic material information, are listed in Table 3. The conductivity information of magnetic material is based on catalogs of each material product. Each material is purchased based on this information, and sample cores are made to measure both $\mathrm{AC}$ and $\mathrm{DC} \mathrm{b}-\mathrm{h}$ curves. Core losses are measured according to variations of both frequency and peak magnetic flux density by the Epstein test method. Fig. 3 shows the measured results of DC $b-h$ curves and a part of core loss data curves. Fig. 3(b) is from one of thirteen samples with different cross-sectional areas [7].

The summarized and compared results for measured core losses are shown in Table 4. The eddy current loss of SMC is lower than that of silicon steel in accordance with representative characteristics of SMC; however, the hysteresis loss is larger. Therefore, total core loss of SMC is larger than that of silicon steel at the condition of the same frequency and flux density under low-frequency domain less than $1 \mathrm{kHz}$. The measured core loss data are used when total core loss is calculated for the analysis models, and the conductivities are used to calculate eddy current loss.

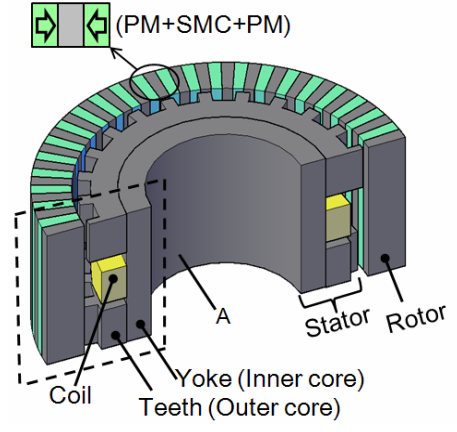

(a)

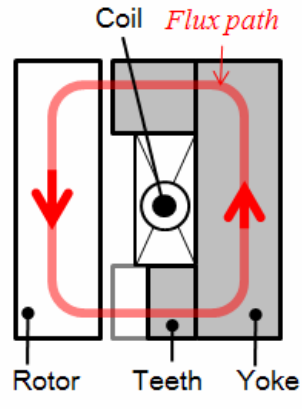

(b)
Fig. 1. Conceptual drawing of one phase in a TFRM; (a) a drawing in section of a TFRM, and (b) flux path on the cross-section of $\mathrm{A}$
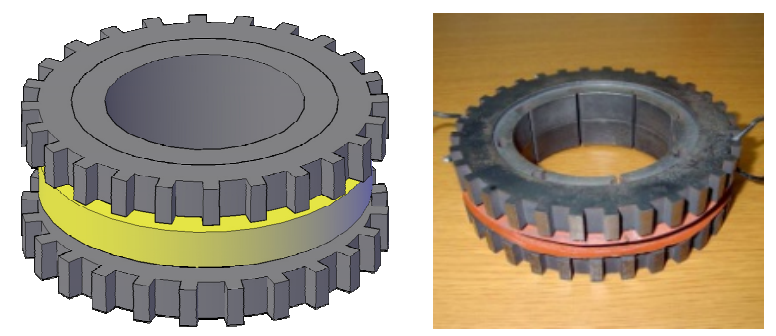

(a) Model SM and its actual object (one-phase core)
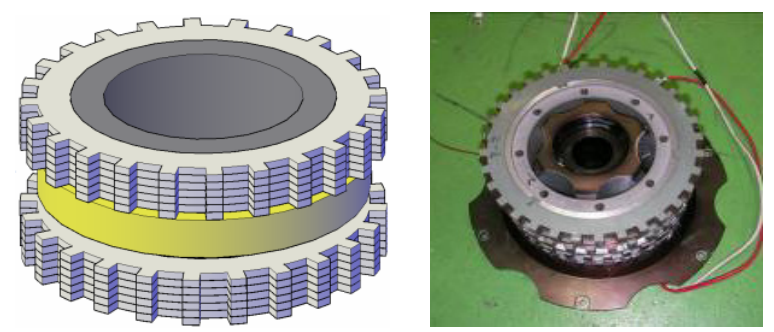

(b) Model SLM (without slits in teeth) and its actual object (two-phase cores)
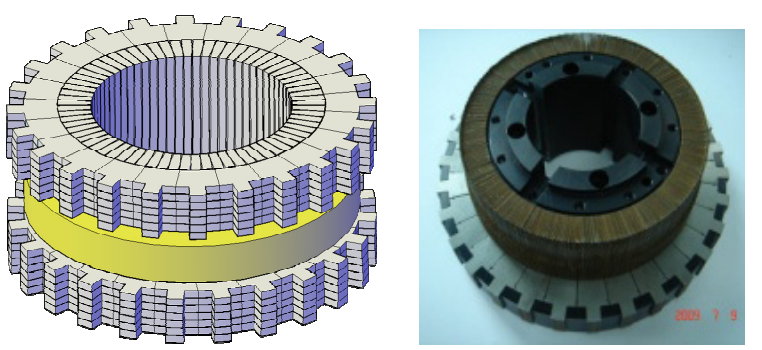

(c) LM model (with slits in teeth) and its actual object (lower teeth + yoke)

Fig. 2. Conceptual drawings and assembly

Table 1. Analysis models

\begin{tabular}{c|c|c}
\hline \multirow{2}{*}{ Analysis model } & \multicolumn{2}{|c}{ Material } \\
\cline { 2 - 3 } & Inner core (yoke) & Outer core (teeth) \\
\hline SM & SMC & SMC \\
\hline SLM & SMC & Silicon steel \\
\hline LM & Silicon steel & Silicon steel \\
\hline
\end{tabular}


Table 2. Main dimensions

\begin{tabular}{c|c}
\hline Rotor outer diameter & $206 \mathrm{~mm}$ \\
\hline Stator outer diameter & $145 \mathrm{~mm}$ \\
\hline Stator inner diameter & $86 \mathrm{~mm}$ \\
\hline Axial length & $112 \mathrm{~mm}$ \\
\hline Air-gap length & $0.5 \mathrm{~mm}$ \\
\hline
\end{tabular}

Table 3. Specifications of an analysis model

\begin{tabular}{c|c|c}
\hline & Quantity & Values and details \\
\hline \multirow{4}{*}{ Stator } & $\begin{array}{c}\text { Material 1 } \\
\text { (conductivity) } \\
\text { Material 2 } \\
\text { (conductivity) }\end{array}$ & $\begin{array}{c}\text { SMC } \\
\text { Laminated silicon steel } \\
(3,000,000 \mathrm{~S} / \mathrm{m})\end{array}$ \\
\cline { 2 - 3 } & No. of phases & 2 \\
\cline { 2 - 3 } & No. of turns & 90 turns/phase \\
\cline { 2 - 3 } & Rated MMF & $620 \mathrm{AT}$ \\
\hline \multirow{4}{*}{ Rotor } & Material & $\begin{array}{c}\text { Core: SMC } \\
\text { PM Nd-Fe-B }\end{array}$ \\
& No. of pole & 60 \\
\cline { 2 - 3 } & Rated speed & $300 \mathrm{rpm}(150 \mathrm{~Hz})$ \\
\cline { 2 - 3 } & & \\
\cline { 2 - 3 }
\end{tabular}

$\mathrm{Br}=$ residual induction; $\mathrm{Hc}=$ coercive force

\section{Magnetic Characteristics}

\subsection{Analysis Results}

Three-dimensional magnetic field FEA was conducted to calculate the electromagnetic parameters and core losses. The commercial software Maxwell was used for the calculation. Fig. 4 shows a three-dimensional analysis model. EMF, inductance, torque, and core losses are obtained by computation results of the time-domain magnetic fields at rated current. The analysis results are shown in Table 5. The value of eddy current loss is normalized based on a value of LM to avoid confusion. Here, because eddy current loss is calculated by a roughly laminated analysis model, the calculated values are not guaranteed; they are used only for comparison.

For both core loss and eddy current loss, SM has the smallest value. This may be because the flux density variation according to time is different from one another, and this is analogized with the torque wave difference (Fig. 5). In other words, the loss of SMC core in SM can be relatively lower because of less harmonic distortion and less peak flux density.

Table 4. Measured eddy current and hysteresis losses

\begin{tabular}{c|c|c|c}
\hline \multirow{2}{*}{ Condition } & $\begin{array}{c}\text { Loss } \\
\text { (W/KG) }\end{array}$ & $\begin{array}{c}\text { SMC (somaloy700) } \\
\text { section: } 6.7 \times 6.7 \mathrm{~mm}^{2}\end{array}$ & $\begin{array}{c}\text { Silicon steel (S23) } \\
\text { Thickness: } 0.5 \mathrm{~mm}\end{array}$ \\
\hline $60 \mathrm{~Hz}$ & Eddy & 0.12 & 0.94 \\
\cline { 2 - 4 } $1.0 \mathrm{~T}$ & Hysteresis & 5.27 & 2.02 \\
\hline $200 \mathrm{~Hz}$ & Eddy & 0.34 & 1.93 \\
$0.5 \mathrm{~T}$ & Hysteresis & 5.68 & 2.57 \\
\hline $400 \mathrm{~Hz}$ & Eddy & 1.35 & 7.72 \\
\cline { 2 - 4 } $0.5 \mathrm{~T}$ & Hysteresis & 11.57 & 4.45 \\
\hline
\end{tabular}

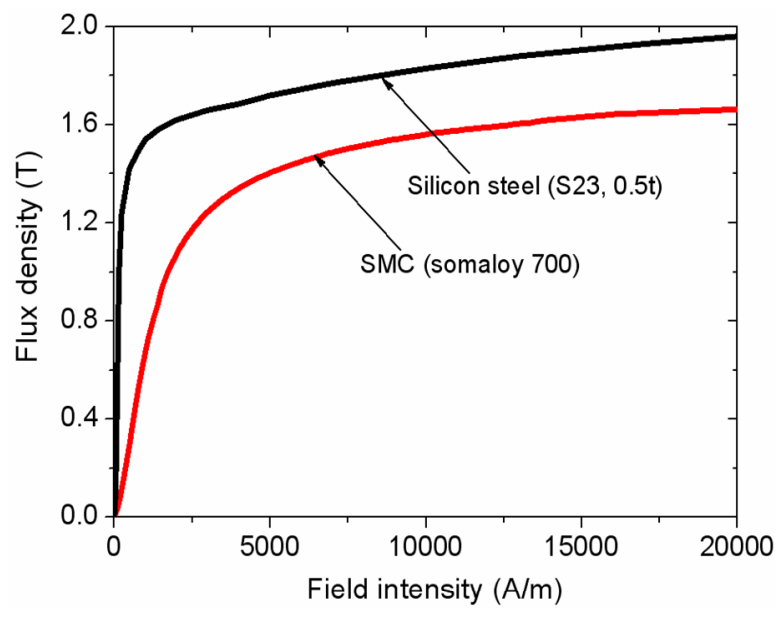

(a) b-h curves

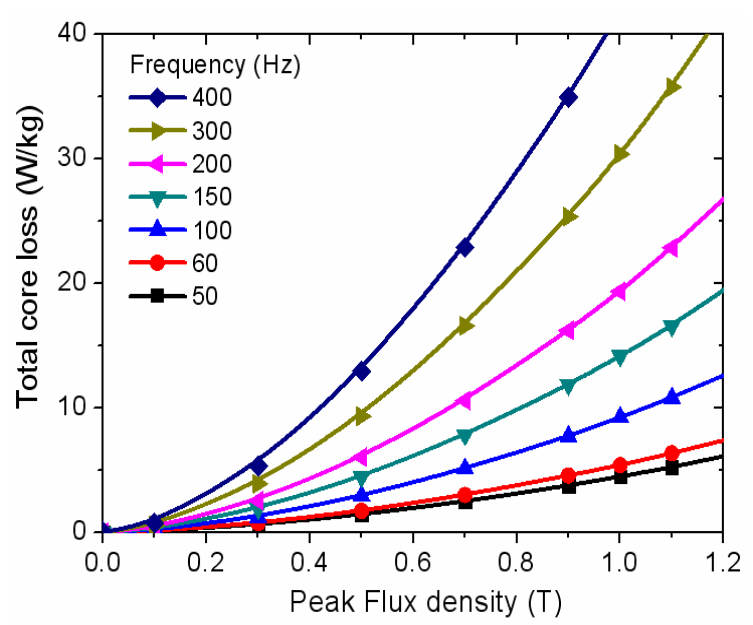

(b) Core losses of SMC core sample $\left(6.7 \times 6.7 \mathrm{~mm}^{2}\right)$

Fig. 3. Magnetic characteristics of core materials

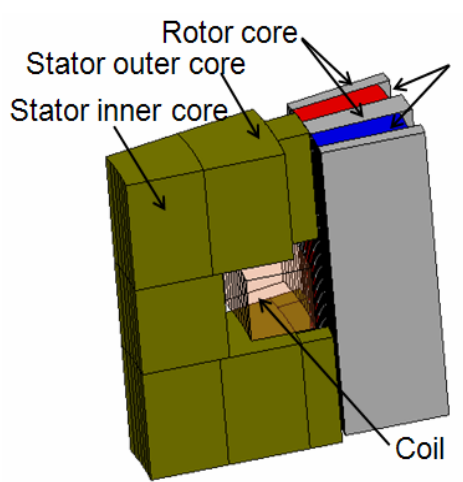

(a)

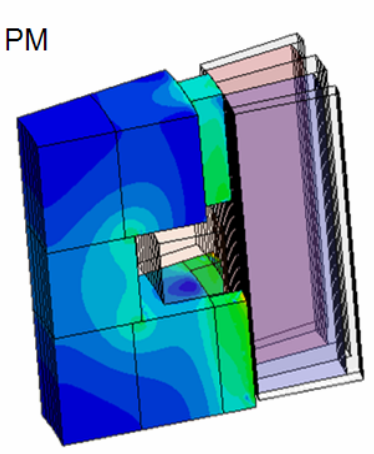

(b)
Fig. 4. Basic analysis model for three-dimensional FEA: (a) configuration of analysis model; (b) flux density distribution in stator 


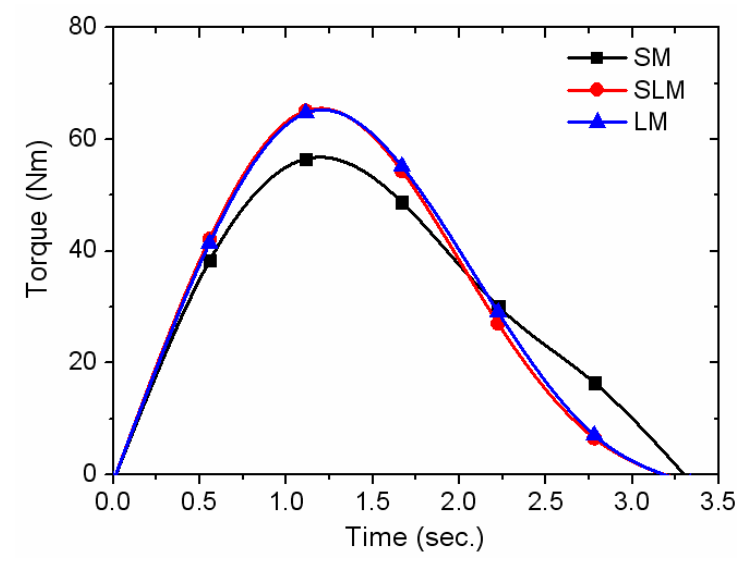

Fig. 5. One-phase torque of three analysis models for rated MMF and rated speed

Table 5. Comparisons of characteristics at rated speed

\begin{tabular}{|c|c|c|c|c|c|c|}
\hline & \multicolumn{2}{|l|}{ Quantity } & Unit & SM & SLM & LM \\
\hline \multirow{5}{*}{$0 \% \mathrm{MMF}$} & \multicolumn{2}{|l|}{ EMF } & $\mathrm{V}$ & 167 & 176 & 179 \\
\hline & \multicolumn{2}{|l|}{ Core loss } & $\mathrm{W}$ & 0.002 & 41.5 & 43.5 \\
\hline & \multirow{3}{*}{$\begin{array}{c}\text { Normalized eddy } \\
\text { current loss } \\
(\mathrm{NC} / \mathrm{C} / \mathrm{S})\end{array}$} & $\mathrm{NC}$ & \multirow{3}{*}{$\%$} & \multirow{3}{*}{0.2} & 56.4 & 100 \\
\hline & & $\mathrm{C}$ & & & 6.7 & 10.8 \\
\hline & & $\mathrm{S}$ & & & 6.5 & 8.1 \\
\hline \multirow{2}{*}{$\begin{array}{l}100 \% \\
\text { MMF }\end{array}$} & $\begin{array}{l}\text { Inductance } \\
\text { (min/max) }\end{array}$ & $\begin{array}{l}\text { Min } \\
\text { Max }\end{array}$ & $\mathrm{mH}$ & $\begin{array}{l}14.3 \\
/ 16.7\end{array}$ & $\begin{array}{l}14.6 \\
/ 17.4\end{array}$ & $\begin{array}{l}15.1 \\
/ 18.2\end{array}$ \\
\hline & \multicolumn{2}{|l|}{ Torque } & $\mathrm{Nm}$ & 31.5 & 32.3 & 32.7 \\
\hline
\end{tabular}

$\mathrm{NC} / \mathrm{C} / \mathrm{S}=$ not considering lamination, considering lamination, and considering lamination and slit, respectively

Depending on considering lamination and slit state, the eddy current loss calculation results are changed. In analysis model, the thickness of layer is $2.5 \mathrm{~mm}$, whereas the real thickness of sheet is $0.5 \mathrm{~mm}$. If the area is reduced five times, the core loss can be reduced a minimum of five times. However, confirming the fact by analysis necessitates considerable time and effort to; therefore, it is verified experimentally.

Based on the analysis results, the comparison of the three models can be expressed by a sign of inequality: (1) parameters related to output power (EMF, torque, inductance): $\mathrm{SM}<\mathrm{LSLM}<\mathrm{LM}$; and (2) eddy current loss and total core losses: $\mathrm{SM}<\mathrm{LSLM}<\mathrm{LLM}$.

\subsection{Experimental Results}

Fig. 6 shows measured EMF according to rotor speed (frequency) variation for all three analysis models, as shown in Fig. 2. The EMF values are normalized because the numbers of turns are different from one another when the three models are fabricated. In the ideal case, the normalized EMF value is 100 at a speed of $600 \mathrm{rpm}$. The SM and LM with slit model EMF values are similar to the ideal case; however, the EMF of SLM without slit is severely nonlinear.

The EMF values are presented in Table 6 for more detailed comparison. The basis of normalized EMF value is

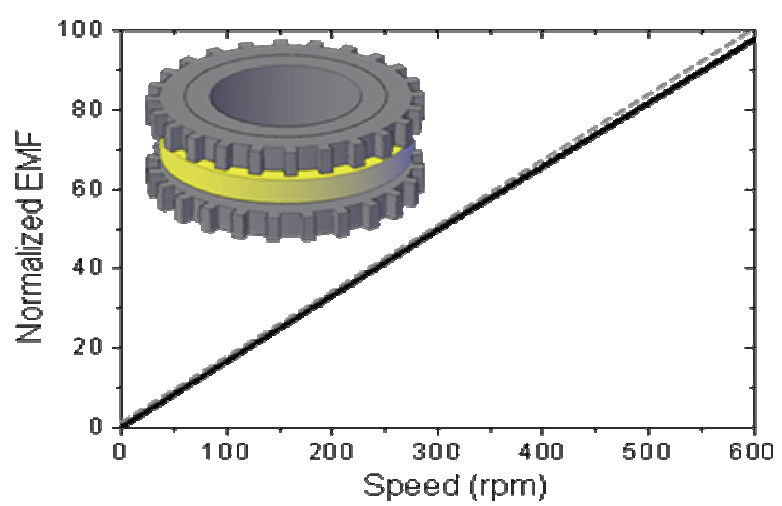

(a) EMF of SM model

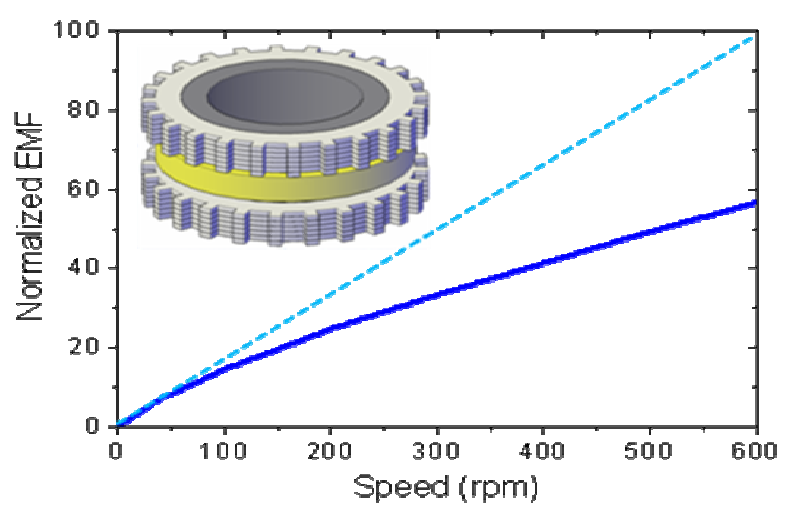

(b) EMF of SLM model (without slit in teeth)

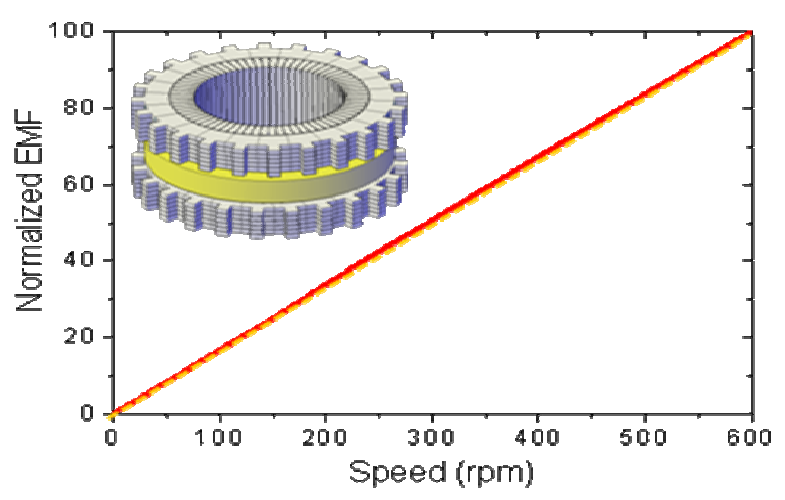

(c) EMF of LM model (with slits in teeth)

Fig. 6. Comparison of measured EMF (dotted line, ideal curve; solid line:, measured curve)

Table 6. Comparison of measured EMF (Normalized Value)

\begin{tabular}{c|c|c|c|c}
\hline \multirow{2}{*}{ Model } & $\begin{array}{c}\text { Stator core } \\
\text { material } \\
\text { (yoke+teeth) }\end{array}$ & $100 \mathrm{rpm}$ & $300 \mathrm{rpm}$ & $600 \mathrm{rpm}$ \\
\hline- & $\begin{array}{c}\text { Ideal case } \\
\text { (assumption) }\end{array}$ & 17 & 50 & 100 \\
\hline SM & - & 17 & 50 & 98 \\
\hline SLM & Without slit & 14 & 34 & 57 \\
\hline \multirow{2}{*}{ LM } & Without slit & 15 & 39 & 66 \\
\cline { 2 - 5 } & With slit & 17 & 50 & 100 \\
\hline
\end{tabular}


the same as in Fig. 6. Whereas the voltage drop is $2 \%$ for $\mathrm{SM}$ at a speed of $600 \mathrm{rpm}$, the other two models using laminated silicon steel have severe voltage drop of $34 \%$ and $43 \%$ because of core losses in stator outer core. This is a very significant result because the core loss is too big, despite the fact that the cores are laminated considering flux path.

To reduce the core losses, slits are formed on outer core in LM. The slit is mechanical, and electrical separation creates smaller eddy current loops. This seems a very effective solution because the voltage drop is 0 in LM with slit. Therefore, the sign of inequality for the total core losses of three models is changed as follows: $\left(2^{\prime}\right)$ estimated total core losses from voltage drop: SLM $>>$ SM $>$ LM (with slit).

\section{Conclusions}

Three-dimensional magnetic field FEA was conducted to calculate the electromagnetic parameters and core losses of the three models. In the analysis, the SM model yielded the best efficiency because of the low core losses. When the two models, SLM and LM, had slits, the eddy current loss was reduced. However, the amount of core loss reduction was too slight to reach the loss of the SM.

The experimental results show different results. When EMF was measured, the SLM and LM without slit exhibited severe voltage drop. When the slits were added to the LM, the voltage drop was eliminated. These results imply that the slits reduce the core losses significantly, and that they affect the core losses more than that predicted in the analysis. The experimental results yield that the performance of LM with slit is better than that of SM after all.

\section{Acknowledgements}

This work was partially supported by the Next Generation Military Battery Research Center program of the Defense Acquisition Program Administration and Agency for Defense Development.

\section{References}

[1] Ji-Young Lee, Ji-Won Kim, Byung-Chul Woo, SangHo Lee, and Jung-Pyo Hong, "Comparison of Magnetic Characteristics according to Stator Core Composition in Transverse Flux Rotary Machine," Proceeding of COMPUMAG 2009

[2] Gene Shane Liew, Nesimi.Etrugrul, Wen Liang Soong, and John Gayler, "An Investigation of Advanced Magnetic Materials for Axial Field Brushless Permanent Magnet Motor Drives for Automotive Applications," IEEE Power Electronics Specialists Con- ference, pp.1-7, June, 2006

[3] Patrick Lemieux, O.Jude Delma, Maxime R.Dubois, and Roderick Guthrie, "Soft Magnetic Composite with Lamellar Particles Application to the Clawpole Transverse Flux Machine with Hybrid Stator," Proceeding of the 2008 International Conference on Electrical Machines, Paper ID 909, 2008

[4] Y.Enomoto, M.Ito, H.Koharagi, R.Masaki, S.Ohiwa, C.Ishihara, and M.Mita, "Evaluation of Experimental Permanent-Magnet Brushless Motor Utilizing New Magnetic Material for Stator Core Teeth," IEEE Trans. on Magnetics, Vol.41, No.11, pp.4304-4308, November 2005

[5] A.G.Jack and B.C.Mecrow, "Calculation of threedimensional electromagnetic fields involving laminar eddy currents," IEE Proceedings, Vol.134, Pt.A, No.8, pp.663-671, September 1987

[6] V.C. Silva, G. Meunier, and A. Foggia, "A 3D FiniteElement Computation of Eddy Currents and Losses in the Stator End Laminations of Large Synchronous Machines," IEEE Trans. on Magnetics, Vol.32, No.3, pp.1569-1572, May 1996

[7] Ji-Young Lee, Ji-Won Kim, Seung-Ryul Moon, JungHwan Chang, Shi-Uk Chung, Do-Hyun Kang, and Jung-Pyo Hong, "Dynamic Characteristic Analysis Considering Core Losses in Transverse Flux Linear Machine with Solid Cores," IEEE Trans. on Magnetics, Vol.45, No.3, pp.1776-1779, March 2009

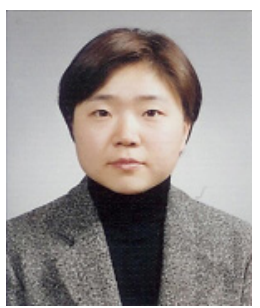

Ji-Young Lee received her B.S., M.S, and Ph.D. degrees in electrical engineering from Changwon National University, Changwon, Korea, in 2000, 2002, and 2006, respectively. Since 2006, she has been with Korea Electrotechnology Research Institute (KERI). Her research interests are focused on the design of electric machines and numerical analysis of electromagnetic field.

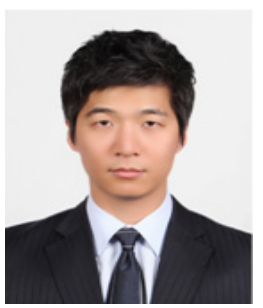

Seung-Ryul Moon received his B.S. degree in computer engineering and M.S. degree in electrical engineering from the Virginia Polytechnic Institute and State University, USA, in 2004 and 2007, respectively. Since 2007, he has been with Korea Electrotechnology Research Institute (KERI) as a researcher. His research interests are high-power dc-dc converters, PWM rectifiers, and motor drives. 


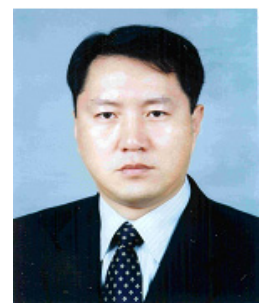

Dae-Hyun Koo received his B.S. and M.S. degrees in electrical engineering from Hanyang University in 1989 and 1991, respectively. Since 1991, he has worked at Korea Electrotechnology Research Institute (KERI). In 2002, he received a Ph.D. degree from Dong-A University. He is currently a director of Mechatronics Research Group, KERI.

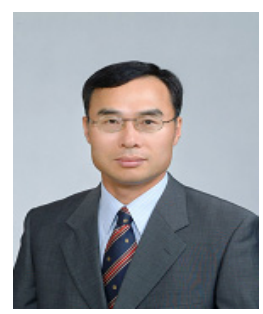

Do-Hyun Kang received B.S. and M.S. degrees from Hanyang University, Seoul, Korea, in 1981 and 1989, respectively, and a Ph.D. degree from the Institute of Electric Machines, Traction and Drives, Technical University Braunschweig, Braunschweig, Germany, in 1996, all in electrical engineering. He was a Design Engineer in the Nuclear Power Plant at Hyundai Construction Company from 1981 to 1986. He is currently the Executive of the Industry Application Research Division of the Korea Electrotechnology Research Institute (KERI). His research interests are magnetic levitation, linear drives, and new electric machines.

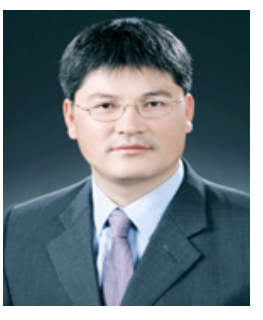

Lee Geun Ho received his B.S. and M.S. degrees in Electrical Engineering and received his Ph.D. degree in Automotive Engineering from the University of Hanyang, Seoul, Korea, in 1992, 1994, and 2010, respectively. From 1994 to 2002, he worked with the LG Industrial System, where he developed an inverter system for elevators. From 2002 to 2010, he was a professor of the Department of Electrical Engineering, Namhae College, GyeongNam Province. Since 2011, he has been a professor of the graduate school of Automotive Engineering, Kookmin University. His research interests are in the areas of advanced control of electrical machines, power electronics, and electric vehicle.

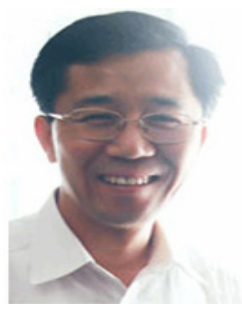

Jung-Pyo Hong received his B.S., M.S., and Ph.D. degrees in electrical engineering from Hanyang University in 1983,1985 , and 1995, respectively. Since 2006 , he has been working as a professor of the Department of Automotive Engineering in Hanyang University. His research interests are the design of electric machines, optimization, vibration and noise reduction, and numerical analysis of electromagnetic field. 